GÊNOVA, L.G. e DUARTE, K.M.R. Padrão de deposição de tecidos protéicos e gordura em cordeiros abatidos precocemente e sua mensuração utilizando a ultra-sonografia. PUBVET, Londrina, V. 5, N. 25, Ed. 172, Art. 1158, 2011.

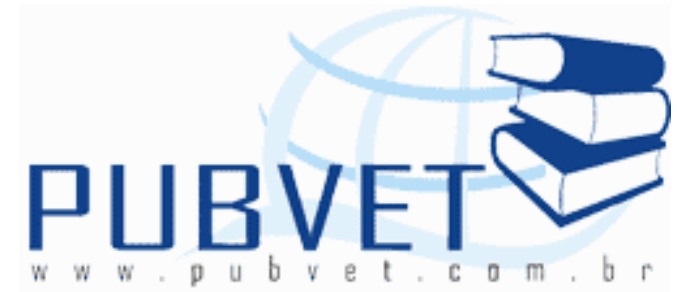

PUBVET, Publicações em Medicina Veterinária e Zootecnia.

\title{
Padrão de deposição de tecidos protéicos e gordura em cordeiros abatidos precocemente e sua mensuração utilizando a ultra-sonografia
}

Laucir Glauco de Gênova ${ }^{1}$ e Keila Maria Roncato Duarte ${ }^{2 *}$

${ }^{1}$ Aluno de Mestrado em Produção Animal Sustentável, Instituto de Zootecnia/APTA - Rua Heitor Penteado, 56 Nova Odessa, SP 13460-000

${ }^{2}$ Docente do curso de Mestrado em Produção Animal Sustentável, Instituto de Zootecnia/APTA *email:keila@iz.sp.gov.br

\section{Resumo}

O manejo do sistema de produção depende do entendimento das relações entre ingestão de alimentos e seus nutrientes, genótipo animal, produção e qualidade de carne. São descritos aqui alguns dos fatores que afetam a eficiência dos alimentos em cordeiros e a composição de tecidos em termos de carne magra, conteúdo de gordura e aspectos de qualidade de carcaça (principalmente conteúdo de gordura e produção de carne). A utilização de técnicas baseadas na análise de imagens (untrasonografia) tem recebido atenção especial e são utilizadas no desenvolvimento de modelos de regressão linear, utilizando o peso vivo e as medidas dos tecidos (espessura de gordura subcutânea, profundidade e área do músculo longissimus) como variáveis independentes

Palavras-chave: cordeiros, ultra-som, carne magra 
GÊNOVA, L.G. e DUARTE, K.M.R. Padrão de deposição de tecidos protéicos e gordura em cordeiros abatidos precocemente e sua mensuração utilizando a ultra-sonografia. PUBVET, Londrina, V. 5, N. 25, Ed. 172, Art. 1158, 2011.

\title{
Standards for protein tissues and fat deposition in lambs slaughtered early and its measurament using ultrasound
}

\begin{abstract}
The management system for meat production depends on the feed intake relations understanding and its nutrients, animal genotype, meat production and quality. In this work, some factors are described affecting food efficiency in lambs as well as the tissue composition of non-fat meat, fat content and carcass quality( fat and meat amount). Image techniques (ultrasound) are being used having special attention with linear regression development models, for live weight and tissue measurements (subcutaneous fat thickness, longissimus muscle deepness and area), as independent variables.
\end{abstract}

Keywords: lambs, ultrasound, non-fat meat

\section{Revisão de literatura}

\section{1-Crescimento e metabolismo}

O crescimento ocorre em ondas, iniciando cranialmente, e posteriormente caudalmente, dos membros distais para o dorso (Palsson,1955). O conceito de maturidade e de considerável importância e para muitos propósitos tem sido definido como o estágio que o corpo do animal contém ao redor de $25 \%$ de gordura. O grau de maturidade pode ser especificado em função do peso vivo (Taylor,1980). Isto significa que para um dado ganho de peso, um animal de grande porte ao peso adulto será menos maturo e magro do que um animal de pequeno porte ao peso adulto. 
GÊNOVA, L.G. e DUARTE, K.M.R. Padrão de deposição de tecidos protéicos e gordura em cordeiros abatidos precocemente e sua mensuração utilizando a ultra-sonografia. PUBVET, Londrina, V. 5, N. 25, Ed. 172, Art. 1158, 2011.

\section{2- Padrão de crescimento}

Em revisão realizada por Black (1983), nota-se uma perda de relação entre a idade do cordeiro e o conteúdo de proteína e gordura corporal, mas observa-se uma intima relação entre conteúdo de proteína, gordura e velocidade de ganho de peso em borregos tosquiados.

Butterfield (1988), detalhou o padrão de desenvolvimento dos órgãos de ovinos; quando a ingestão de alimentos não é restringida , a proporção de crescimento após o nascimento é maior para o cérebro, intestino, fígado, coração e músculos que para o peso corporal e os depósitos de gordura contribuem em menor proporção do ganho de peso corporal (Black,1983; Butterfield, 1988).

Quando a ingestão de alimentos e restringida, particularmente no período anterior a $30 \%$ da maturidade, a proporção de alguns órgãos com intestino, fígado e músculos podem ter suas dimensões reduzidas (Tulloh et al.1986).

\section{3- Utilização da energia do alimento para mantença.}

Como conceito de energia para mantença, temos a energia metabolizável gasta para a produção de calor; nos ovinos devido a produção de lã, não existe um requerimento que a composição do corpo permaneça inalterado, causando ganho ou perda de gordura e proteína do corpo, além disto existe também consideráveis outras variações possivelmente genéticas, na eficiência do alimento utilizado para mantença (Herd et al., 1993).

\section{4- Utilização da energia do alimento para crescimento.}

Um dos métodos envolvidos para medir a deposição de proteína e energia gasta em cordeiros ingerindo diferentes quantidades com a mesma alimentação foi estudada por Oddy, 1999. Este sugere que a eficiência de deposito de proteína no 
GÊNOVA, L.G. e DUARTE, K.M.R. Padrão de deposição de tecidos protéicos e gordura em cordeiros abatidos precocemente e sua mensuração utilizando a ultra-sonografia. PUBVET, Londrina, V. 5, N. 25, Ed. 172, Art. 1158, 2011.

músculo depende da taxa de síntese de proteínas, menos a degradação desta, sendo esta a taxa de acréscimo de proteína.

Uma aplicação prática seria o desenvolvimento de genótipos de cordeiros com alta eficiência de utilização de energia para acréscimo de proteína. Dado que o calor produzido é proporcional ao ganho de massa e síntese de proteínas a identificação de genótipos que diferem em síntese de proteínas associada a calor de produção tem um potencial para reduzir significativamente a quantidade de alimento para conseguir um determinado peso e composição corporal, uma conseqüência não desejável desta estratégia, é que animais com baixas taxas de degradação de proteínas musculares também tem diminuída a proteólise pós morte, o que resulta no endurecimento da carne (McDonagh et al., 1999).

\section{5- Metabolismo da gordura.}

O metabolismo da gordura e sua deposição são controlados por fatores hormonais, nutricionais e dependem da idade do animal e seu aporte de energia.

A taxa de deposição de gordura é relacionada a quantidade de energia avaliável em excesso dos requerimentos para mantença e ganho de peso, do que mudanças metabólicas no tecido adiposo (Sainz e Wolff, 1990).

\section{6- Efeitos do genótipo.}

Depois da desmama, os nutrientes são oriundos da ingestão e digestão de alimentos formados de celulose. A habilidade do cordeiro para ingerir e digerir estes alimentos são ambos influenciados por caracteres ineridos (genes).

\section{7- Crescimento e composição dos tecidos.}

O padrão de deposição de proteína e gordura em ovinos de diferentes genótipos são similares na maturidade; com exceção da raça Texel que tem aproximadamente $2 \%$ a mais de proteína e menos gordura (Kempster et al., 
GÊNOVA, L.G. e DUARTE, K.M.R. Padrão de deposição de tecidos protéicos e gordura em cordeiros abatidos precocemente e sua mensuração utilizando a ultra-sonografia. PUBVET, Londrina, V. 5, N. 25, Ed. 172, Art. 1158, 2011.

1987), e na ocorrência de uma mutação chamada "Callipyge" que ocorre nos músculos traseiros, aumentando seu peso em $40 \%$ aos seis meses de idade em relação aos animais controle (Koohmaraie et al., 1995). Isto ocorre como conseqüência de hipertrofia das fibras musculares.

\section{8- Efeito da dieta na composição de ganho de peso.}

De acordo com o estágio de maturidade a relação de contribuição de proteína e gordura, quanto a energia retida, varia com o acréscimo de ingestão de alimentos.

Quanto mais jovem o cordeiro, mais terá acréscimos de proteínas no corpo e relativamente alta proporção de energia retida em proteína. A quantidade de ganho em gordura e determinada após os gastos de mantença e ganhos de proteína, aumentando com a ingestão de alimentos em excesso da mantença. Taxas de ganho em gordura também aumentam quando o cordeiro alcança aproximadamente $70 \%$ da maturidade.

\section{9- Ganho de peso compensatório.}

Ovinos freqüentemente enfrentam períodos de restrição alimentar, seguidos por períodos de compensação alimentar, podendo ocorrer também pelos efeitos de doenças parasitárias; este ganho de peso é mais rápido e eficiente que ganhos normais e é chamado ganho compensatório. A composição do ganho compensatório em ovinos no período de realimentação contém mais proteínas do que em ovinos em crescimento continuo (Hegarty et al., 1999).

\section{0- Uso da ultrasonografia para avaliação de carcaças de ovinos.}

Trabalhos tem sido publicados em metodologias de estimativa de composição carcaça em ovinos segundo Alliston (1983), Simm (1987), Topel e Kauffman (1988), e Stanfort et al., (1998). Destacando-se as metodologias: 1) 
GÊNOVA, L.G. e DUARTE, K.M.R. Padrão de deposição de tecidos protéicos e gordura em cordeiros abatidos precocemente e sua mensuração utilizando a ultra-sonografia. PUBVET, Londrina, V. 5, N. 25, Ed. 172, Art. 1158, 2011.

ultrasonografia em tempo real, 2) ressonância magnética nuclear, 3) tomografia computadorizada e 4)análise por ativação de nêutrons. Sendo todas estas consideradas promissoras para o desenvolvimento de trabalhos de investigação cientifica uma vez que todas não são destrutivas e não dolorosas para os animais (Kempster, 1984; Simm, 1987; Wells (1991).

$\mathrm{Na}$ escolha da metodologia levamos em consideração a precisão para uma determinada utilização e seu custo benefício, além disso deve ser prática robusta móvel e não deve prejudicar o desempenho dos animais (Allen, 1990). A ultrasonografia em tempo real apresenta uma relação custo / beneficio apropriada para uso no campo, como também na investigação cientifica, Alliston (1983), Kempster (1984) e Wells (1991).

Kirton (1989), considerou a ultrasonografia em tempo real como a tecnologia com maior aplicação na avaliação de carcaças. Esta tecnologia dispõe de equipamentos robustos, portáteis e de utilização simples, sendo utilizada para aplicação em bovinos (Simn, 1983; Houghton e Turlington, 1992), em suínos (Gresham et al., 1994), em ovinos (Silva et al., 1994; Cadavez et al., 2000; Santos et al., 2000) e em caprinos (Delfa et al. 1998; Azevedo et al., 1999; Cadavez et al., 2002b).

Segundo Augusto et al. 2000, existem uma grande variedade de aparelhos de ultrasonografia. Em geral, o aparelho e constituído por um monitor, onde a imagem e observada, e um ou mais transdutores onde os pulsos de ultrasom são emitidos e captados. No monitor existem controles para regular a intensidade das ondas de som captadas pelo transdutor, alterar a amplificação dos ecos de retorno e determinar a profundidade da imagem visualizada na tela. A maioria dos modelos também apresenta um teclado para anotação de dados e tem funções para obtenção de medidas (Cartee, 1995; Green, 1996). No monitor os ecos de retorno são representados por pontos cuja profundidade depende do 
GÊNOVA, L.G. e DUARTE, K.M.R. Padrão de deposição de tecidos protéicos e gordura em cordeiros abatidos precocemente e sua mensuração utilizando a ultra-sonografia. PUBVET, Londrina, V. 5, N. 25, Ed. 172, Art. 1158, 2011.

tempo de retorno destes ecos. Existe uma escala de cor cinza para cada ponto correspondendo a amplitude ou a forca do eco de retorno. Os ecos de pouca intensidade são representados como imagens de cor negra, os de media intensidade em tons de cinza e os de alta intensidade em branco (Herring e Bjornton, 1989; Nyland, Mattoon e Wisner, 1995).

O transdutor é o instrumento mais importante para a avaliação ultrasonográfica. Assim a escolha correta do tipo e freqüência é essencial para uma melhor visualização da imagem. Existem vários tipos e modelos que variam de acordo com a área a ser examinada. O transdutor e caracterizado por conter em seu interior cristais pizoelétricos. Os cristais possuem a capacidade de emitir pulsos de ultrasom, através de estímulo elétrico, em diferentes freqüências de comprimento de onda. Alguns transdutores são multifrequenciais, mas apenas um grupo de cristais é selecionado por vez. Os tipos mais comuns de transdutores são os lineares, convexos, micro-convexo e setorial (Barr, 1990). O transdutor linear e eletrônico é composto por múltiplos cristais que são dispostos em linha, dentro da porção cranial do transdutor, a desvantagem deste transdutor é necessitar de uma grande área de contato com a pele do animal, não sendo este um entrave a ultrasonografia de carcaças em ovinos, adaptandose muito bem a este fim.

A freqüência que o transdutor emite é definida como o número de ondas de ultrasom que são repetidas por segundo, estas repetições são denominadas ciclos. A freqüência e expressa em milhões de ciclos por segundo, sendo o pequeno comprimento de onda essencial para uma boa resolução de imagem. A freqüência e o comprimento de onda são inversamente proporcionais se a velocidade média permanecer constante. A velocidade do som é independente da freqüência e quase constante (1540 m/seg) nos tecidos moles (Wells, 1969; Barr, 1990). Contudo, se a onda de ultra-som encontra osso ou gás ocorre uma reflexão, 
GÊNOVA, L.G. e DUARTE, K.M.R. Padrão de deposição de tecidos protéicos e gordura em cordeiros abatidos precocemente e sua mensuração utilizando a ultra-sonografia. PUBVET, Londrina, V. 5, N. 25, Ed. 172, Art. 1158, 2011.

devido a combinação de uma mudança brusca na velocidade ou na densidade da interface tecido-osso ou tecido-gas, ocorrendo significativa redução da velocidade (Herring e Bjornton, 1989; Cartee, 1995; Farrow, 1996). A profundidade com que a onda de ultra-som penetra em tecidos moles é diretamente relacionada com a freqüência utilizada.

As ondas de ultra-som de maior freqüência são mais atenuadas que as de maior freqüência. Isto significa que melhorando a resolução, com o aumento da freqüência, ocorrerá uma menor penetração da onda de ultra-som. As ondas de ultra-som e os ecos de retorno são atenuados a medida que atravessam os tecidos. Quanto mais distante a interface que reflete o eco, mais fraco será o eco de retorno (Farrow, 1992).

A imagem ultra-sonografica e baseada no principio de pulso de eco. Isto significa que as ondas de ultra-som são produzidas pelo transdutor em pulsos contínuos. A imagem e formada pelos ecos, que retornam dos tecidos pelo transdutor depois de cada pulso. Existe um tempo adequado para que todos os ecos retornem, antes do transdutor emitir outro pulso.

O transdutor emite o pulso em menos de $1 \%$ do tempo de ação, sendo que nos 99\% restantes aguarda e capta o retorno dos ecos (Curry, Dowdey e Murry, 1990).

Quando os cristais pulsam, aproximadamente dois ou três comprimentos de ondas de ultra-som são emitidos em cada pulso. Um transdutor de alta freqüência emite comprimento de onda de ultra-som curto, conseqüentemente terá um pulso mais curto que o transdutor de menor freqüência (Herring e Bjorton, 1985). 
GÊNOVA, L.G. e DUARTE, K.M.R. Padrão de deposição de tecidos protéicos e gordura em cordeiros abatidos precocemente e sua mensuração utilizando a ultra-sonografia. PUBVET, Londrina, V. 5, N. 25, Ed. 172, Art. 1158, 2011.

Quanto ao modo de disposição dos ecos existem Três modos denominados A, B E M; Os modos B e M são os mais utilizados em medicina veterinária (Barr, 1990; Cartee, 1995).

O modo B - brilho - utiliza múltiplas ondas de ultra-som, sendo os ecos de retorno representados como pontos no monitor. Brilho ou escala de cinza são proporcionais a amplitude do eco de retorno e a posição dos pontos corresponde a profundidade na qual o eco e originado (Park et al. 1981).

Aplicações principais da ultrasonografia em tempo real na ciência animal como metodologia de estimativa in vivo da composição da carcaça; 1) em programas de seleção de reprodutores para características de qualidade da carcaça e da carne (Brethour, 1992; Lo et al., 1992; Kemp et al., 2002; 2) manejo alimentar e treinamento de avaliação da condição corporal (Delfa et al.,1991; Domecq et al.,1995, bem como na validação da metodologia de avaliação da condição corporal (Schwager-Suter et al., 2000; Cadavez et al., 2002a; Bueno et al., 2003; 3) na definição do ponto ótimo de abate (Kempster e Owen,1982; Fuller et al.,1990 ; Houghton e Turlington, 1992; Stanford et al., 1998).

Para utilização da técnica de ultrasonografia em tempo real nas medidas dos tecidos das carcaças é necessário um estudo prévio para elucidar a relação entre estas e as medidas homologas efetuadas na carcaça. Quanto menor esta diferença maior e a exatidão da técnica (Cochran e Cox, 1957), medindo tão próximos são os valores individuais estimados dos valores reais. Quanto menor for a diferença entre o valor real e o valor estimado, ou seja, quanto mais próximo de zero e o desvio, maior é a exatidão. A precisão da técnica e definida como a repetibilidade entre medidas sucessivas (Cochran e Cox, 1957), ou seja, mede tão próximas são entre si as medidas sucessivas. Neste caso medidas sucessivas dos tecidos são efetuadas por ultrasonografia em tempo real e quanto menor é a diferença entre o valor médio e o valor real maior e a precisão. 
GÊNOVA, L.G. e DUARTE, K.M.R. Padrão de deposição de tecidos protéicos e gordura em cordeiros abatidos precocemente e sua mensuração utilizando a ultra-sonografia. PUBVET, Londrina, V. 5, N. 25, Ed. 172, Art. 1158, 2011.

Analisando os conceitos de exatidão e precisão, podemos concluir que a ultrasonografia em tempo real pode apresentar uma precisão elevada e ao mesmo tempo ter baixa exatidão. Ou seja, se a diferença entre as medidas de ultrasonografia em tempo real e as medidas homologas da carcaça for grande, apesar desta medida apresentar uma elevada precisão, apresentará, com certeza, baixa exatidão, pelo que os resultados obtidos com uma técnica precisa podem ser parcialmente ou totalmente falsos.

Cuidados na coleta de imagens na ultrasonografia em tempo real (UTR) devem ser tomados, pois a qualidade destas e de suma importância para sua correta interpretação (Hopkins et al., 1996; Simm, 1983). A precisão das medidas de UTR depende dos fatores que induzem variação na interpretação das imagens, cuja facilidade de interpretação esta diretamente ligada a qualidade das mesmas. A localização e identificação do ponto anatômico de referencia sobre o qual se efetua a medida e suas distorções pelo posicionamento dos animais também vão influenciar a coleta das imagens (Silva, 2001).

A interpretação das imagens também e passível de erros (Simm,1983; Hopkins et al.,1996) observando-se diferenças entre operadores com treinamento diferenciado (Purchas e Beach, 1981; McLaren et al.,1991), mas também entre operadores masters (Purchas e Beach, 1981; Perkins et al.,1992b; Robinson et al.,1992; Herring et., 1994; Moeller e Christian,1998). McLaren et al.(1991) também verificaram que o operador representou uma maior fonte de variação na interpretação das imagens do que na sua obtenção, pelo que estes autores consideraram que deve ser dada grande importância a interpretação das imagens. Por outro lado, Young et al.(1992) observaram, em ovinos, que a captura das imagens representou uma maior fonte de variação do que a sua interpretação, uma vez que as medidas repetidas na mesma imagem apresentaram repetibilidade superior as medidas repetidas em imagens 
GÊNOVA, L.G. e DUARTE, K.M.R. Padrão de deposição de tecidos protéicos e gordura em cordeiros abatidos precocemente e sua mensuração utilizando a ultra-sonografia. PUBVET, Londrina, V. 5, N. 25, Ed. 172, Art. 1158, 2011.

diferentes, na medição do músculo longissimus $(0,98$ vs 0,74$)$, mas também na espessura da gordura subcutânea $(0,95$ vs 0,78$)$.

A influência do operador também e influenciada pela localização e características dos tecidos a serem escaneados, segundo Yong et al., (1992), em ovinos e de Waldner et al., (1992) em bovinos estes verificaram que repetibilidade entre operadores experientes e inexperientes foi semelhante na mensuração da medida da espessura da gordura subcutânea. Entretanto os operadores experientes obtiveram melhores resultados na mensuração da profundidade do músculo longissimus. Isto explica a facilidade de obter uma boa imagem para medir a espessura da gordura subcutânea, e a dificuldade de obtenção de uma boa imagem para medir a área ou a profundidade do músculo longissimus, requerendo tempo, experiência e bom conhecimento de anatomia topográfica.

Além da exatidão das medidas de UTR serem dependentes destes fatores que condicionam a precisão da técnica, são também afetadas pelos fatores de erros de medição das medidas homologas das carcaças, sendo estes ocasionados por: 1) erros de leitura; 2) erros provocados pela remoção de parte dos tecidos durante a retirada do couro, especialmente na medição da gordura subcutânea; 3) erros provocados pela deformação dos tecidos durante o processo de abate, refrigeração e corte da carcaça e 4 ) erros resultantes da realização das medidas em localizações anatômicas diferentes das medidas efetuadas por UTR.

Vários trabalhos estudaram a relação entre as medidas dos tecidos realizadas por UTR e as medidas homologas efetuadas na carcaça em ovinos (Kempster et al., 1982a ; Young et al.,1992; Ber et al.,1997; Cadavez et al.,1999; Santos et al.,2000; Silva,2001; Cadavez et al.,2002), através do calculo de coeficientes de correlação linear entre as medidas de UTR e as medidas homologas efetuadas na carcaça, designando-os por precisão ou estabelecendo métodos de regressão linear entre as medidas de UTR e as homologas da carcaça. A dificuldade de 
GÊNOVA, L.G. e DUARTE, K.M.R. Padrão de deposição de tecidos protéicos e gordura em cordeiros abatidos precocemente e sua mensuração utilizando a ultra-sonografia. PUBVET, Londrina, V. 5, N. 25, Ed. 172, Art. 1158, 2011.

comparação entre os resultados destes trabalhos reside na elevada variação no material animal, local a ser efetuada a medida, na experiência dos operadores, nos equipamentos e na freqüência da sonda utilizada.

Quanto a freqüência da sonda utilizada esta varia de 3,5 a 7,5 mhz, para medir os mesmos tecidos. Assim, em uma determinada profundidade o aumento de freqüência limita a resolução porque a amplitude do eco e diminuída e para a mesma freqüência a resolução é também deteriorada, aumentando a profundidade de observação (Wells,1991). A freqüência a utilizar será dependente da magnitude do tecido a medir e a sua localização no corpo do animal. Em tecidos superficiais com a gordura subcutânea os melhores resultados serão com uma sonda de freqüência mais elevada. Por outro lado em tecidos de maior magnitude e localização mais interna, como por exemplo o músculo longissimus, serão de esperar melhores resultados com sondas de freqüência mais baixa.

O local a ser efetuada as medidas foi estudada por Fernandes et al.,1998, encontrando a melhor localização para medida de espessura de gordura entre a $12^{a}$ e $13^{a}$ costela $(r 2=0.92, P<0.001)$, em borregos da raça Manchego; Silva et al., 2005, também encontrou na região da $13^{a}$ costela o melhor preditor de gordura subcutânea. Trabalhos mais recentes como de Teixeira et al., 2006, utilizaram para espessura de gordura entre a $12^{a}$ e $13^{a}$ costela e entre a $3^{a}$ e $4^{a}$ vértebra lombar na raça Churra Galega Bragançana e Ripoll et al., 2009, entre $10^{a}$ e $12^{a}$ costela, $12^{a}$ e $13^{a}$ costela, $1^{a}$ e $2^{a}$ vértebra lombar e $3^{a}$ e $4^{a}$ vértebra lombar, avaliando medidas de músculos e gordura na raça Tenasco.

\section{Considerações finais}

Como conceito de maturidade podemos definir como estágio que o corpo do animal contém ao redor de $25 \%$ de gordura, sendo este dependente de seu porte 
GÊNOVA, L.G. e DUARTE, K.M.R. Padrão de deposição de tecidos protéicos e gordura em cordeiros abatidos precocemente e sua mensuração utilizando a ultra-sonografia. PUBVET, Londrina, V. 5, N. 25, Ed. 172, Art. 1158, 2011.

ao peso adulto. Quando é alcançada cerca de $70 \%$ da maturidade aumentam as taxas de ganho em gordura, sendo esta também dependente da raça estudada.

Uma ferramenta eficaz para mensuração in vivo de deposição de proteína e gorduras corporais é a ultra-sonografia, contanto que seja realizado uma padronização dos conhecimentos técnicos e utilização de sondas apropriadas para o uso em ovinos.

\section{Referências bibliográficas:}

Allen, P., 1990. Measuring body compositiom in live meat animals. In Reducing fat in meat animals. Eds. J.D. Wood e A. V. Fischer. Elsevier Applied Science, pp. 201-254.

Alliston , J.C., 1983. Evaluation of carcass quality in live animal. In Sheep production. Ed. W. Haresign. Butterworths, pp. 75-95.

Augusto, A. Q.; Pachaly, J. R. Princípios físicos da ultra-sonografia - Revisão bibliográfica. Arq. Ciên. Vet. Zool., UNIPAR, 3(1): p. 61-65, 2000.

Azevedo, J. M. T, Silva, S. J. C. R. e Gomes, E.D., 1999. Estimativa da composição da carcaça de bodes maduros a partir de medidas obtidas por ultrasons. Revista Protuguesa da Zootecnia, 2 (VI): 55-67.

Barr, F. Principles of diagnostic ultrasound. In Diagnostic ultrasound in the dog and cat. London: Blackwell Scientific Publications, 1990. p. 1-20

Berg, E.P., Neary, M.K., Forrest, J.C. Thomas, D.L. e Kauffman, R.G., 1997. Evaluation of electronic Technology to assess lamb carcass composition. Journal of Animal Science, 75 (9): 2433-2444.

Black, J. L. (1983) Growth and development of lambs. In Haresign, W. (Ed) Sheep Production. Butterworths, London, pp. 21-58.

Brethour, J. R., 1992. The repeatability and accuracy of ultrasound in measuring backfat of cattle. Journal of Animal Science, 70: 1039-1044.

Bueno, M. S., Matos, S. Cadavez, V., Pereira, E., Rodrigues, S., Batista, S., Delfa, R. e Teixeira, A., 2003. Repetibilidad y reproducibilidade de lãs medidas de tejidos in vivo efectuadas com ultrasonidos em ovejas adultas y sus correlaciones com La condición corporal. $X$ Jornadas Sobre Producción Animal, Zaragoza. ITEA. 142-144.

Butterfield, R.M. (1988) New Concepts of Sheep Growth. Griffin Press, Netley, South Austrália.

Cadavez, V. A. P.; 2004, ultra sonografia para valorizar in vivo e exvivo carcaças de ovinos, estudos nas raças Churra Galega Bragançana e Suffolk, Universidade de Trás-os-Montes e Alto Douro, Vila Real, 2004. 
Cadavez, V., Matos, S., Rodrigues, S., Pereira, E., Delfa, R. e Teixeira, A., 2002a. Repetibilidade e reprodutibilidade das medidas in vivo de tecidos efectuadas por ultrasons em ovelhas adultas. XII Congresso de Zootecnia, Vila Real. APEZ. pp. 581-584.

Cadavez, V., Teixeira, A., Delfa, R e Rodrigues , S., 2000. Utilizacion de ultrasonidos y el peso vivo para La prediccion in vivo de La composición de la canal em corderos. XXV Jornadas Cientificas y IV Internacionales de La Sociedad Española de Ovinotecnia y Caprinotecnia, Teruel. pp 165-168.

Cadavez, V., Teixeira, A., Delfa, R. e Pereira, E., 1999. Precision de los ultrasonidos (sondas de 5 y 7,5 MHz) em la determinacion del spessor de La grasa subcutânea y la profundidad del $\mathrm{m}$. longissimus dorsi in vivo y em la canal. VIII Jornadas Sobre Producción Animal, Zaragoza. Pp 119121.

Cadavez, V.A.P., Rodrigues, S., Pereira, E., Delfa, R. e Teixeira. A, 2002. Predicción de la composición de la canal de cabritos por ultrasonografia in vivo, ITEA, 98A(1): 39-50.

Cartee, R. E. The physics of ultrasound. In: CARTEE et at. Practical veterinary ultrasound. Philadelphia: Willians \& Wilkins, 1995. p. 1-8.

Curry, T. S. III: Dowdey, J. E.; Murry, R. C. Jr. Christensen's physic of diagnostic radiology. 4. ed., Philadelphia: Lea \& Febiger, 1990. 323 p.

Delfa, R., Teixeira, A. e Colomer-Rocher, F., 1991. Cold carcass weight, fat thickness, C measurement and longissimus dorsi depth for predicting the carcass composition of rasa Aragonesa ewes with different body condition score. Options Méditerranéenes - Série Séminaires, pp. 19-24.

Delfa, R., Teixeira, A., e Gonzalez, C., 1998. Body weight and ultrasound as predictors of carcass quality and fat partition in adult goats. Revista Protuguesa de Zootecnia, 2: 1-16

Domecq, J.J., Skidmore, A. L., Lloyd, J.W. e Kaneene, J. B., 1995. Validation of body condition scores with ultrasound measurements of subcutaneous fat of dairy cows. Journal of Dairy Science, 78(10): 2308-2313.

Farrow, C. S. How Ultrasound works. In: Green, R. W. Small animal ultrasound. Philadelphia: Lippincoll-Raven, 1996. p. 7-27.

Farrow, C. S. Ultra talk: beginners guide to the language of ultrasound. Veterinary radiology \& ultrasound. Raleigh. V.33, n. 1, p. 33-31, 1992.

Fernández, C., Garcia, A., Vergara, H. e Gallego, L. 1998. Using Ultrasound to determine fat thickness and longissimus dorsi área on Manchego lambs of different live weight. Small Ruminant Research, 27(2): 159-165.

Fuller, M. F., Fowler, P. A., McNeil, G. e Foster, M.A., 1990. Body Composition: the prediction and accuracy of new methods and their suitability for longitudinal studies. Proceedings of the Nutrition Society, 49: 423-436.

Green, R. W. The veterinary ultrasound. In Small animal ultrasound. Philadelphia: LippincottRaven, 1996. p. 1-5. 
Gresham, J. D., McPeake, S. R., Bernard, J. K., Riemann, M. J., Wyatt, R.W. e Henderson, H. H., 1994. Prediction of live and carcass characteristics of market hogs by use of a single longitudinal ultrasonic scan. Journal of Animal Science, 72: 1409-1416.

Hegarty, R.S., Neutze, S.A. and Oddy, V.H. (1999) Effects of protein and energy supply on the growth and carcass composition of lambs from differing nutritional histories. Journal of Agricultural Science, Cambridge 1321, 361-375.

Herd, R.M., Oddy, V.H. and Lee, G.J. (1993) Effect of divergentselection for weaning weight on liveweight and wool growth responses to feed intake in Merino ewes. Australian Journal of Experimental Agriculture 33, 699-705.

Herring, W. O., Miller, D.C., Bertrand, J.K. e Benyshek, L.L., 1994. Evaluation of machinetechnician, and interpreter effects on ultrasonic measures of backfat and longissimus muscle área in beef catle. Journal of Animal Science, 72: 2216-2226.

Herring, D.S.; Bjornton, G. Physics, Facts, and artifacts of diagnostic ultrasound. The veterinary clinics of North America: small animal practice. Philadelphia: W. B. Saunders. V. 15. p. 11071122, 1985.

Herring, D.S.; Bjornton, G. Physics, facts, and artifacts of diagnostic ultrasound. Seminars in medicine and surgery(small animal). Philadelphia, v.4, n. 1. p. 2-12, 1989.

Hopkins, D.L., Hall, D.G. e Luff, A.F., 1996. Lamb carcass. 3. Describing changes in carcasses of growing lambs using real-time ultrasound and the use of these measurements for estimating the yield of saleable meat. Australian Journal of Experimental Agriculture, 36(1): 37-43.

Houghton, P. L. e Turlington , L. M., 1992. Aplication of ultrasound for feeding and finishing animals: A review. Journal of Animal Science, 70: 930-941.

Kemp, J.D., Herring, W. O. e Kaiser, C. J. , 2002. Genetic and enviromental parameters for steer ultrasound and carcass traits. Jornal of Animal Science, 80: 1489-1496.

Kempster, A.J., Croston, D., Guy, D>R. and Jones, D.W. (1987) Growth and carcass characteristics of cross bred lambs by tem sire breeds, compared at the same estimated subcutaneous fat proportion. Animal Production 44, 39-49.

Kempster, A.J., 1984. Cost-benefit analyses of in vivo estimates of body composition in meat animals. In In vivo measurements of body composition in meat animals. Ed. D. Lister. Elsevier Applied Science, pp. 191-203.

Kempster, A.J., e Owen, M. G., 1982. A note on the accuracy of na ultrasonic technique for selecting cattle of different breeds for slaughter ate qual fatness. Animal Production, 32: 113-115.

Kempster, A.J., Arnall, D., Alliston, J.C. e Backer, J.D., 1982a. Na evaluation of two ultrasonic machines (Scanogram and Danscanner) for predicting the body composition of live sheep. Animal Production, 34: 249-255.

Kirton, A. H., 1989. Current methods of on-line carcass evaluation . Journal of Animal Science, 67: $2155-2163$. 
Koohmaraie, M., Shackleford, S.D., Wheeler, T.L., Lonergan, S.M. and Doumit, M.E. (1995) A muscle hypertrophy condition in Lamb (callipyge): characterization on effects on muscle growth and meat quality traits. Journal of Animal Science 73, 3596-3607.

Lo, I. I., McLaren, D. G., McKeith, F. K., Fernando, R. L. e Novakofski, J., 1992. Genetic analyses of growth, real-time ultrasound, carcass, and pork quality traits in Duroc and Landrace pigs. II. Heritabilities and correlations. Journal of animal science, 70: 2357-2396.

McDonagh, M.B., Fernandez, C. and Oddy, V.H. (1999) Hind-Limb protein metabolism and calpain system activity influence post-mortem changes in meat qualityof Lamb. Meat Science 52, 9-18.

McLaren, D.G., Novakofski, J., Parrett, D.F., Lo, L.L., Singh, S.D., Neumann, K.R. e McKeith, F.K., 1991. A study of operator effects on ultrasonic measures of fat depth and longissimus muscle área in catle, sheep and pigs. Journal of Animal Science, 69: 54-66.

Moeller, S.J. e Christian, L.L., 1998. Evaluation of real-time ultrasonic measurements of back fat and loin muscle área in swine using multiple statistical analysis procedures. Journal of Animal Science, 76: 2503-2514.

Nyland, T. G.; Mattoon, J. S. Wisner, E. R. Physical principles, instrumentation, and safety of diagnostic ultrasound. In: Nyland, T. G.: Mattoon J. S. Veterinary diagnostic ultrasound. Philadelphia: W. B. Saunders, 1995. p. 3-18.

Oddy, V.H. (1999) Protein metabolism and nutrition in farm animals: na overview. In: Lobley, G.E., White, A. and MacRae, J.C. (eds) Protein Metabolism and Nutrition, Proceedings of the VIIIth International Symposium on Protein Metabolism and Nutrition. EAAP Publication No. 96, Wageningen Pers, The Netherlands, pp. 7-23.

Oddy, V.H., Speck, P.A., Warren, H.M. and Wynn, P.C. (1995) Protein metabolism in lambs from lines divergently selected for weaning weight. Journal of Agricultural Science, Cambridge 124, 129-137.

Palsson, H. (1955) Conformation and body composition. In: Hammond, J. (ed.) Progress in the Physiology of Farm Animals, Vol. 2 Butterworths, London, pp. 430-542.

Park, R. D. et al B- mode gray-scale ultrasound: imaging artifacts and interpretation principles. Veterinary radiology. Raleigh, v. 22. p. 204-210,1981.

Perkins, T.L., Green, R.D., Hamlin, K.E., Shepard, H.H. e Miller, G.J., 1992b.Ultrasonic prediction of carcass merit in beef cattle: Evaluation of technician effectson ultrasonic estimates of carcass fat thickness and longissimus muscle area. Journal of Animal Science, 70: 2758-2765.

Purchas, R.W. e Beach, A.D., 1981. Between-operator repeatability of fat depth measurements made on live sheep and lambs with an ultrasonic probe. New Zealand Journal of Experimental Agriculture, 9: 213-220.

Ripoll, G., Joy, M., Alvarez-Rodriguez,J., Sans, A. and Teixeira, A. (2009) Estimation of light Lamb carcass composition by in vivo real-time ultrasonography at four anatomical localions. Journal of Animal Science 87, pg 1455-1463.

Robinson, D.L., Mcdonald, C.A., Hammond, K e Turner, J.W., 1992. Live animal measurement of carcass traits by ultrasound: assessment and accuracy of sonographers. Journal of Animal Science, 70: 1667-1676. 
Sainz, R.D. and Wolff, J.E. (1990) Development of a dynamic, mechanistic modelo $f$ Lamb metabolism and growth. Animal Production 51, 535-549.

Santos, V.C., Silva, S. J. C. R., Azevedo, J. e Gomes, E. D., 2000. Estimativa da composição da carcaça de ovinos da raça Ilê-de-France a partir de medidas obtidas por ultrasons ao nível da $13^{a}$ vértebra dorsal e entre as $3^{a}$ e $4^{a}$ vértebras lombares. Revista Portuguesa de Zootecnia, VII(2): 91-104.

Schwager-Suter, R., Stricker, C., Erdin, D. e Kunzi, N. (2000). Relationship Between body condition scores and ultrasound measurements of subcutaneous and m.longissimus dorsi in dairy cows differing in size and type. Animal Science, 71: 465-470

Silva, S.R., Gomes, M.J., Dias-da-Silva, A., Gil, L.F. and Azevedo, J.M.T. (2005) Estimation in vivo of the body and carcass chemical composition of growing lambs by real-time ultrasonography. Journal of Animal Science 83, pg 350-357.

Silva, S.J.C.R., 2001. Composição das carcaças e dos depósitos internos de gordura de ovelhas da raça Churra da Terra Quente. Tese de Doutoramento, Depatamento de Zootecnia, Universidade de Trás-os-Montes e Alto Douro. Vila Real, pp.296.

Silva, S., Azevedo, J. e Monteiro, A., 1994. Estimativa da composição da carcaça de borregos a partir de medidas por ultrasons ao nível da $13^{a}$ vértebra dorsal e entre as $3^{a}$ e $4^{a}$ vértebra lombares. Revista Portuguesa de Zootecnia, I(1): 77-82.

Simm, G., 1987. carcass evaluation in sheep breding programmes. In New Techniquesin Sheep Production. Eds.I. F. Maral e J. F. Owen. Butterworths. London, pp. 125-144.

Simm, G., 1983. The use of ultrasound to predict the carcass composition of live catle A review. Animal Breeding Abstracts, 51: 853-875.

Stanford, K., Jones, S. D. M. e Price, M.A., 1998. Methods of predicting lab carcass composition: A review. Small Ruminant Research, 29: 241-254.

Taylor, StC.S. (1980) genetic size-scaling rules in animal growth. Animal Production 30, 160-165.

Teixeira, A., Matos, S., Rodrigues, S., Delfa, R. and Cadavez, V. (2006) In vivo estimation of Lamb carcass composition by real-time ultrasonography. Meat Science, vol. 74, Issue 2 pages 289-295.

Topel, D. e Kauffman, R., 1988. Live animal and carcass composition measurement. In Designing Foods: Animal product options in the marketplace. Ed. N. A. Press. Washington, D. C., pp. 258272.

Tulloh, N.M. Brimblecombe, H. and Deniss, C. (1986) The effect of severe nutritional deprivation in early post-natal life: tissue and cellular responses during subsequent growth of lambs to the age of 4 months. Journal of Agricultural Science, Cambridge 106, 341-350.

Waldner, D.N., Dikeman, M.E., Schalles, R.R., Oson, W.G., Houghton, P.L., Unruh, J.A. e Corah, L.R., 1992. Validation of real-time ultrasound technology for predicting fat thicknesses, longissimus muscle áreas, and composition of Brangus bulls from 4 months to 2 years of age. Journal of Animal Science, 70: 3044-3054. 
Wells, P.N.T., 1991. The description of animal form and function. Livestock Production Science, 27: $19-33$.

Wells, P.N.T., 1991. The physical principles of ultrasonic diagnosis. London: Academic Press, 1969. $38 \mathrm{p}$.

Young, M.J., Deaker, J.M. e Logan, C.M., 1992. Factors affecting repeatability oftissue depth determination by real-time ultrasound in sheep. Proceedings of the New Zealand Society of Animal Production, 52: 37-39. 